\title{
Efeito da adiposidade corporal elevada na aptidão física relacionada à saúde de
}

\section{escolares da rede pública de ensino}

\author{
Effect of high body adiposity on physical fitmess related to health of schools in the public teaching
} network

Efecto de la alta adiposidad corporal sobre la aptitud física relacionada con la salud de las escuelas de la red de enseñanza pública

Jean Cesar Andrade de Souza

ORCID: https://orcid.org/0000-0002-0313-0279 Universidade Federal de São Carlos, Brasil

E-mail: jeancesar.andrade@gmail.com

Arthur Tromboni Francischette

ORCID: https://orcid.org/0000-0003-2163-5884

Centro Universitário de Votuporanga, Brasil E-mail: arthur95_area@hotmail.com

Amanda Cunha dos Santos

ORCID: https://orcid.org/0000-0002-3715-5263

Centro Universitário de Votuporanga, Brasil

E-mail: amandacunha_san@hotmail.com

Taiele Rodrigues da Silva Cruz

ORCID: https://orcid.org/0000-0001-6937-5028

Centro Universitário de Votuporanga, Brasil E-mail: taielesilv@hotmail.com

Leonardo Gasques Trevisan Costa

ORCID: https://orcid.org/0000-0001-7711-205X Universidade Federal do Vale do São Francisco, Brasil E-mail: leonardo.gasques@univasf.edu.br

Caciane Dallemole Souza

ORCID: https://orcid.org/0000-0002-1487-5989

Centro Universitário de Votuporanga, Brasil E-mail: caciane.d@gmail.com

Valter Mariano dos Santos Júnior ORCID: https://orcid.org/0000-0002-2650-9441

Centro Universitário de Votuporanga, Brasil

E-mail: profvaltermariano@gmail.com

\begin{abstract}
Resumo
Introdução: Concomitantemente ao avanço mecânico e tecnológico observa-se na sociedade contemporânea a emersão de aspectos sedentários e preferência por dietas hipercalóricas que juntas favorecem a instalação da obesidade e declínio da aptidão física, principalmente em crianças e adolescentes em idade escolar. Objetivo: Avaliar e verificar o efeito da adiposidade corporal na aptidão física relacionada à saúde de escolares da rede pública de ensino. Método: A amostra foi composta por 363 escolares de ambos os sexos, com idade entre 9 e 17 anos, selecionados por voluntariedade em escolas do município de Votuporanga/SP. Todos os voluntários foram submetidos a avaliações antropométricas: peso, estatura e dobras cutâneas (tricipital e panturrilha); e testes motores referentes às capacidades físicas: flexibilidade (FLEX), resistência muscular localizada (RML) e aptidão cardiorrespiratória (ApC). Posteriormente foram calculados o Índice de Massa Corporal (IMC) e percentual de gordura (\%G). Para análise dos dados foi utilizado o software estatístico SPSS 22.0, adotando significância de p>0,05. Resultados: Foram verificadas correlações negativas entre \% $\mathrm{G}$ e as capacidades físicas FLEX $(\mathrm{r}=-0,147 ; \mathrm{p}=0,045)$ e RML $(\mathrm{r}=-0,328 ; \mathrm{p}=0,000)$ nas meninas, e RML ( $\mathrm{r}=-0,330 ; \mathrm{p}=0,000)$ e ApC $(\mathrm{r}=-0,475 ; \mathrm{p}=0,000)$ nos meninos. Conclusão: Quanto maior o \%G menor o desempenho dos escolares nos testes motores referentes as capacidades físicas estudadas, portanto, a obesidade nesses indivíduos se caracteriza como fator preponderante à baixa aptidão física, que por sua vez, pode favorecer o surgimento de comorbidades, prejudicando os processos de desenvolvimento humano e início da vida adulta.
\end{abstract}

Palavras-chave: Adiposidade; Adolescentes; Aptidão física; Crianças. 
Research, Society and Development, v. 10, n. 3, e52310313680, 2021

(CC BY 4.0) | ISSN 2525-3409 | DOI: http://dx.doi.org/10.33448/rsd-v10i3.13680

\begin{abstract}
Introduction: Concomitantly with the mechanical and technological advance, contemporary society shows an emergence of sedentary aspects and a preference for high-calorie diets that together favor the installation of obesity and decline in physical fitness, especially in school-age children and adolescents. Objective: To evaluate and verify the effect of body adiposity on physical fitness related to the health of public school students. Method: The sample consisted of 363 students of both sexes, aged between 9 and 17 years old, selected voluntarily in schools in the municipality of Votuporanga/SP. All volunteers were found to be anthropometric assessments: weight, height and skin folds (tricipital and calf); and motor tests defined as physical resources: flexibility (FLEX), localized muscle resistance (RML) and cardiorespiratory fitness (ApC). Subsequently, the Body Mass Index (BMI) and fat percentage $(\% \mathrm{~F})$ were calculated. To analyze the data used in the statistical software SPSS 22.0, adopting a significance of $\mathrm{p}>0.05$. Results: There were negative correlations between $\% \mathrm{~F}$ and physical resources FLEX $(\mathrm{r}=-0.147 ; \mathrm{p}=0.045)$ and RML ( $\mathrm{r}=-0.328 ; \mathrm{p}=0.000)$ in girls, and RML ( $\mathrm{r}=-0.330 ; \mathrm{p}=0.000)$ and ApC $(\mathrm{r}=-0.475 ; \mathrm{p}=0.000)$ in boys. Conclusion: The higher the $\% \mathrm{~F}$, the lower the performance of students in motor tests regarding the studied physical capacities, therefore, obesity in these individuals is characterized as a preponderant factor to low physical fitness, which, in turn, can favor the emergence of comorbidities, impairing the processes human development and early adulthood.
\end{abstract}

Keywords: Adiposity; Adolescents; Physical fitness; Children.

\title{
Resumen
}

Introducción: Paralelamente a los avances mecánicos y tecnológicos, en la sociedad contemporánea surgen aspectos sedentarios y una preferencia por las dietas hipercalóricos que en conjunto favorecen la aparición de la obesidad y el deterioro de la forma física, especialmente en niños en edad escolar y adolescentes. Objetivo: Evaluar y verificar el efecto de la adiposidad corporal sobre la aptitud física relacionada con la salud de los escolares en el sistema escolar público. Método: La muestra estuvo conformada por 363 escolares de ambos sexos, con edades entre 9 y 17 años, seleccionados voluntariamente en escuelas del municipio de Votuporanga/SP. Se encontró que todos los voluntarios fueron evaluados antropométricamente: peso, altura y pliegues cutáneos (tricipital y pantorrilla); y pruebas motoras definidas como recursos físicos: flexibilidad (FLEX), resistencia muscular localizada (RML) y aptitud cardiorrespiratoria (ApC). Posteriormente, se calculó el Índice de Masa Corporal (IMC) y el porcentaje de grasa $(\% \mathrm{G})$. Analizar los datos utilizados en el software estadístico SPSS 22.0, adoptando una significancia de $\mathrm{p}>0,05$. Resultados: Hubo correlaciones negativas entre \%G y recursos físicos FLEX ( $\mathrm{r}=-0,147 ; \mathrm{p}=0,045)$ y RML ( $\mathrm{r}=-0,328$; $\mathrm{p}=0,000)$ en niñas y $\mathrm{RML}(\mathrm{r}=-0,330 ; \mathrm{p}=0,000)$ y $\mathrm{ApC}(\mathrm{r}=-0,475 ; \mathrm{p}=0,000)$ en niños. Conclusión: A mayor \% G, menor es el desempeño de los escolares en las pruebas motoras con respecto a las capacidades físicas estudiadas, por lo que la obesidad en estos individuos se caracteriza como un factor preponderante para la baja aptitud física, lo que, a su vez, puede favorecer la aparición de comorbilidades, perjudicando los procesos de desarrollo humano y adultez temprana.

Palabras clave: Adiposidad; Adolescentes; Aptitud física; Niños.

\section{Introduçãa}

Concomitantemente ao avanço mecânico e tecnológico observa-se cada vez mais na sociedade contemporânea a emersão de aspectos sedentários e preferência por dietas hipercalóricas que associadas favorecem a instalação da obesidade e declínio da aptidão física, provocando consequências graves à saúde das pessoas, principalmente em crianças e adolescentes (Paiva \& Costa, 2015; Bermejo-Cantarero et al., 2017; Freitas et al., 2020).

Caracterizando-se como uma das principais causas patológicas da modernidade, a obesidade tem na diminuição do tempo em atividade física de intensidade moderada à vigorosa e, ingestão de alimentos hipercalóricos ricos em carboidratos e lipídeos, seus principais determinantes (Mendonça \& Anjos, 2004; Fermino et al., 2010; Costa et al., 2020; Nascimento et al., 2021). Por conseguinte, este quadro patológico de origem multifatorial pode intensificar abruptamente os riscos de alterações fisiológicas características da síndrome metabólica (Faria et al., 2014; Oliveira \& Guedes, 2016), culminando no aumento das taxas de morbidade e mortalidade (Zimmermann \& Matheus, 2017).

Adicionalmente, o acúmulo excessivo de gordura corporal evidenciado nesta patologia, combinado ao estado inflamatório crônico de baixo grau, se correlacionam ao polimorfismo gênico, a inibição de hormônios hipotalâmicos e disfunções na sinalização de adipocinas que juntas resultam em um balanço energético positivo, cenário alarmante e favorável a evolução precoce de quadros clínicos severos que na infância podem prejudicar o desenvolvimento humano e transição para 
a vida adulta (Pereira-Lancha et al., 2012; Paes et al., 2015; Oliveira \& Guedes, 2016; Zawadzki et al., 2019; Nascimento et al., 2021).

Simultaneamente ao aumento das taxas de obesidade em todo o mundo, a aptidão física, especificamente as capacidades físicas associadas à saúde dos indivíduos, tem padecido (Petroski et al., 2011; Lopes et al., 2019). A manutenção de longos períodos de exposição à inatividade física propicia o adiantamento de efeitos fisiológicos deletérios que podem antecipar estados de velhice (Meneguci et al., 2015). No entanto, o incentivo à prática regular de exercícios físicos e aquisição de hábitos alimentares saudáveis, ainda na infância, podem prevenir ou diminuir o impacto desta patologia na vida das pessoas (Costa et al., 2020).

Nesse contexto, por se configurarem como componentes essenciais à saúde e desempenho esportivo, a análise da flexibilidade, força e resistência muscular, resistência cardiorrespiratória e composição corporal por meio de parâmetros antropométricos e testes motores podem servir como acompanhamento do estado de saúde de crianças e adolescentes, uma vez que os dados provenientes destas análises possibilitam a detecção de aspectos físicos, morfológicos e patológicos prejudiciais à saúde desses indivíduos (Paes et al., 2015; Mello et al., 2015; Oliveira et al., 2017; Nahas, 2017; Castro et al., 2018).

Sob essa ótica é relevante avaliar e identificar fatores que possam interferir ou se apresentar como coeficientes benéficos ou prejudiciais à evolução dos hábitos humanos, afinal mesmo que diversos estudos realizados nos últimos anos discorram sobre esta temática e, apesar do reconhecimento de sua importância, nota-se ainda um aumento das taxas de obesidade infantil e inatividade física (Zimmermann \& Matheus, 2017).

Logo, o objetivo deste estudo foi avaliar e verificar o efeito da adiposidade corporal com a aptidão física relacionada à saúde de escolares da rede pública de ensino do município de Votuporanga/SP.

\section{Metodologia}

\subsection{Caracterização da Pesquisa}

Este estudo caracterizou-se como uma pesquisa de abordagem quantitativa, do tipo transversal (Thomas et al., 2012; Pereira et al., 2018). Segundo o censo da Secretaria Municipal da Educação de Votuporanga/SP (2016), acrescido da projeção de matriculas do Instituto Brasileiro de Geografia e Estatística (2017), em 2017 o município possuía 12.474 escolares em nível fundamental e médio. O cálculo do tamanho amostral ideal foi realizado através da fórmula de Santos (2017), obtendo-se o quantitativo de 363 escolares, sendo 175 meninos e 188 meninas, com idade entre 9 à 17 anos, regularmente matriculados em instituições da rede pública de ensino do município.

Os critérios de inclusão adotados foram: (a) comparecer na data, horário (das 8 às 11 horas da manhã) e local de avaliação previamente agendado (4 unidades escolares de ensino público de Votuporanga/SP equipadas com quadra poliesportiva e salas climatizadas); (b) apresentar o Termo de Consentimento Livre e Esclarecido (TCLE) assinado pelos responsáveis; (c) mostrar-se interessados em participar, por voluntariedade, do estudo através da assinatura do Termo de Assentimento Livre e Esclarecido (TALE); (d) estar em bom estado de saúde, não possuindo quaisquer tipos de limitações clínicas e/ou funcionais (doenças cardíacas, respiratórias, osteomusculares, imunológicas, genéticas ou endócrinas). Os critérios de exclusão adotados foram: (a) não autorização dos responsáveis; (b) não credenciamento dos escolares na realização das avaliações em qualquer momento da coleta de dados; (c) não estar condizente com os critérios de inclusão descritos anteriormente. 
Research, Society and Development, v. 10, n. 3, e52310313680, 2021

(CC BY 4.0) | ISSN 2525-3409 | DOI: http://dx.doi.org/10.33448/rsd-v10i3.13680

\subsection{1. Ética}

Todos os procedimentos utilizados no desenvolvimento desta pesquisa seguiram as normas que regulamentam a pesquisa com seres humanos (Resolução 466/12 do Conselho Nacional de Saúde), havendo sido submetido e aprovado pelo Comitê de Ética e Pesquisa em Seres Humanos do Centro Universitário de Votuporanga - UNIFEV (Parecer n ${ }^{\circ} .113 .774$ ).

\subsection{Procedimentos Experimentais}

Todas as avaliações ocorreram em instituições de ensino públicas autorizadas pelos órgãos gestores (Departamentos e Secretarias de Educação). Antes do início da coleta de dados os alunos receberam instruções sobre os procedimentos à serem realizados. Todos os testes e medidas foram realizados pelo mesmo avaliador e, os dados anotados em planilha individual. A avaliação da composição corporal e da aptidão física relacionado à saúde respeitou a sequência proposta pelo Projeto Esporte Brasil - PROESP-BR (Gaya \& Gaya, 2016).

Para avaliação da composição corporal foram utilizadas: balança antropométrica digital modelo W200 (Welmy ${ }^{\circledR}$, Santa Barbara D’Oeste, Brasil), com precisão de 100 gramas e capacidade de 200 quilos; estadiômetro portátil Personal Caprice ES2060 (Sanny ${ }^{\circledR}$, São Bernardo do Campo, Brasil), com escala de precisão de 0,1cm; e adipômetro modelo Classic AD1007 (Sanny ${ }^{\circledR}$, São Bernardo do Campo, Brasil), com precisão em milímetros, realizando-se três aferições para cada dobra cutânea.

O Índice de Massa Corporal (IMC) foi determinado pela razão do peso pela estatura ao quadrado (IMC = peso/estatura $\left.{ }^{2}\right)$. A mensuração do percentual de gordura $(\% \mathrm{G})$ foi realizado por meio da equação proposta por Slaughter et al. (1988) para crianças e adolescentes de 8 à 18 anos, a qual se vale das espessuras das dobras cutâneas tricipital (TR) e panturrilha (PT) para predição da adiposidade corporal $\left[\left(\underline{\% G \text { Meninos }}=0,735^{*}(\Sigma 2)+1\right) ;(\underline{\% \text { Meninas }}=0,610 *(\Sigma 2)+5,1)\right]$, onde $\Sigma 2$ é a somatória das dobras cutâneas TR e PT.

A flexibilidade (FLEX) foi determinada por meio do teste de Sentar-e-Alcançar, no qual o avaliado deveria posicionar os calcanhares nas extremidades de uma fita adesiva de 30 centímetros colocada perpendicularmente na marca dos 38 centímetros de uma fita métrica fixada no solo, com os joelhos estendidos e mãos sobrepostas e, quando autorizado deveria inclinar-se estendendo simultaneamente as mãos à frente, o mais distante possível. Foram realizadas duas tentativas, sendo anotada a de melhor desempenho (Gaya \& Gaya, 2016).

Já a resistência muscular localizada (RML) foi determinada pelo teste motor de repetições abdominais-sit up em 1 minuto. Para iniciar tal protocolo, o avaliado deveria demonstrar total compreensão do teste, bem como posicionar-se em decúbito dorsal com os joelhos semiflexionados ( $45^{\circ}$ graus) e, braços cruzados sobre o tórax. Durante o protocolo o avaliador permaneceu segurando com as mãos os tornozelos do estudante, fixando-os ao solo. Foi considerado uma repetição completa quando o avaliado se retirou da posição de inércia através de uma flexão de tronco, encostou os cotovelos na parte anterior da coxa e, retorno a posição inicial (Gaya \& Gaya, 2016).

Por fim, a aptidão cardiorrespiratória (ApC) foi determinada pelo teste de corrida/caminhada de 6 minutos realizado em quadra poliesportiva delimitada nas extremidades por cones, e nas bordas laterais por marcações no solo referentes a distância percorrida em metros. Ao sinal do avaliador os escolares transcorreram a maior distância possível durante esses minutos. Ao segundo sinal sonoro, o avaliado foi instruído a parar e aguardar até que fosse registrada a distância percorrida em metros (Gaya \& Gaya, 2016). 


\subsection{Análise de dados}

Os dados obtidos foram digitados em planilhas do programa Microsoft Excel 2013 e as análises estatísticas foram processadas utilizando o software IBM SPSS ${ }^{\circledR}$ (Statistical Package for the Social Sciences), versão 22.0 for Windows (SPSS Inc., Chicago, Estados Unidos). Inicialmente, a distribuição dos dados foi analisada pelo teste Kolmogorov-Smirnov. Após a confirmação da normalidade, foram efetuadas análises descritivas (média, desvio padrão, frequência). O teste $t$ de Student foi adotado para verificar possíveis diferenças entre meninos e meninas nas variáveis estudadas (idade, estatura, peso, IMC, dobras cutâneas TR e PT, \%G, FLEX, RML, ApC). Posteriormente, foram verificadas correlações entre idade, peso, IMC e \% G com o desempenho motor nos testes de aptidão física para cada capacidade física (FLEX, RML, ApC) por meio do teste de Pearson. A magnitude das correlações foi avaliada qualitativamente de acordo com Hopkins et al. (2009) (de 0,9 à $1=$ quase perfeita; de 0,7 à $0,9=$ muito alta; de 0,5 à $0,7=$ alta; de 0,3 à $0,5=$ moderada; de 0,1 à $0,3=$ baixa; $<0,1=$ muito baixa). Os resultados foram expressos em média e desvio padrão. A significância adotada foi de 5\%, portanto foram consideradas significativas as comparações e correlações cujo p<0,05.

\section{Resultados}

A caracterização da amostra e os dados referentes as variáveis de idade, composição corporal e capacidades físicas são apresentadas na tabela 1. Os resultados indicam diferenças significativas entre os sexos em estatura $(\mathrm{p}<0,010)$, peso $(\mathrm{p}<0,037)$ e ApC $(\mathrm{p}<0,010)$ com os meninos apresentando escores médios maiores que as meninas, e em dobra cutânea PT $(\mathrm{p}<0,012)$ e \%G $(\mathrm{p}<0,018)$ onde ocorre um cenário inverso com as meninas apresentando escores médios mais elevados. $\mathrm{O}$ mesmo não foi observado em idade, IMC, Dobra Cutânea TR, FLEX e RML.

Tabela 1. Comparação das médias \pm desvio padrão das variáveis de idade, composição corporal e capacidades físicas entre sexos.

\begin{tabular}{|c|c|c|c|c|}
\hline & Total $(n=363)$ & Meninas $(n=188)$ & Meninos $(n=175)$ & $p$ \\
\hline Idade (anos) & $13,60 \pm 2,3$ & $13,48 \pm 2,4$ & $13,72 \pm 2,2$ & 0,148 \\
\hline Estatura (m) & $1,58 \pm 11,9$ & $1,55 \pm 0,08$ & $1,61 \pm 0,13$ & $\mathbf{0 , 0 1 0} * *$ \\
\hline Peso (kg) & $51,63 \pm 14,1$ & $49,19 \pm 12,2$ & $54,38 \pm 15,4$ & $\mathbf{0 , 0 3 7} *$ \\
\hline $\operatorname{IMC}\left(\mathrm{kg} / \mathrm{m}^{2}\right)$ & $20,35 \pm 3,8$ & $20,23 \pm 3,7$ & $20,52 \pm 3,8$ & 0,975 \\
\hline Dobra Cutânea TR (mm) & $13,47 \pm 6,6$ & $15,48 \pm 6,0$ & $11,15 \pm 6,5$ & 0,122 \\
\hline Dobra Cutânea PT (mm) & $13,24 \pm 6,7$ & $14,75 \pm 6,0$ & $11,65 \pm 7,2$ & $0,012 * *$ \\
\hline$\% \mathbf{G}$ & $20,73 \pm 8,7$ & $23,57 \pm 6,7$ & $17,74 \pm 9,5$ & $\mathbf{0 , 0 1 8} * *$ \\
\hline FLEX (cm) & $32,33 \pm 9,9$ & $34,58 \pm 9,3$ & $29,85 \pm 9,8$ & 0,851 \\
\hline RML (rep) & $25,01 \pm 8,1$ & $21,72 \pm 6,9$ & $28,51 \pm 7,6$ & 0,384 \\
\hline ApC (m) & $922,59 \pm 180,7$ & $824,72 \pm 109,0$ & $1024,00 \pm 186,0$ & $0,010 * *$ \\
\hline
\end{tabular}

Legenda: IMC: Índice de Massa Corporal; TR: Tricipital; PT: Panturrilha; \%G: Percentual de Gordura.

*Correlação significativa para $\mathrm{p} \leq 0,05 ; * *$ Correlação significativa para $\mathrm{p} \leq 0,01$.

Fonte: Autores.

De modo geral, analisando os dados referentes a tabela 2 na qual estão expressos os valores das correlações das variáveis estudadas (idade, peso, IMC, \%G) com os testes motores da aptidão física relacionada à saúde (FLEX, RML, ApC) de acordo com o sexo, notou-se que apenas o \% $\mathrm{G}(\mathrm{r}=-0,147$; $\mathrm{p}<0,045)$ de meninas apresentou correlação significativa com a FLEX. Em continuação, foi verificado correlação entre RML e as variáveis de idade $(r=0,239 ; p<0,000)$ e \%G (r=-0,420; $\mathrm{p}<0,000)$ na amostra total, assim como nos meninos ( $\mathrm{r}=0,398$; $<<0,000$ para idade; e r=-0,330; $<<0,000$ para \%G). Já no grupo feminino a RML se correlacionou com peso ( $r=-0,164$; $p<0,025)$, IMC ( $r=-0,181 ; p<0,013)$ e \%G $(r=-0,328 ; p<0,000)$. Além 
disso, na amostra total a ApC se correlacionou com a idade ( $r=0,269 ; \mathrm{p}<0,000)$, peso $(\mathrm{r}=0,179 ; \mathrm{p}<0,001)$ e \%G $(\mathrm{r}=-0,474$; $\mathrm{p}<0,000)$. Nos meninos a ApC correlacionou-se com a idade ( $r=0,507 ; \mathrm{p}<0,000)$, peso $(r=0,184 ; \mathrm{p}<0,015), \mathrm{IMC}(\mathrm{r}=-0,070$; $\mathrm{p}<0,033)$ e \% $\mathrm{G}(\mathrm{r}=-0,474 ; \mathrm{p}<0,000)$, por fim, nas meninas está correlação somente foi observada com o IMC ( $\mathrm{r}=-0,150$; $\mathrm{p}<0,041)$.

Tabela 2. Análise da correlação entre capacidades físicas e variáveis antropométricas de acordo com o sexo.

\begin{tabular}{|c|c|c|c|c|c|c|c|c|c|}
\hline & \multirow{2}{*}{$\begin{array}{l}\text { Capacidade } \\
\text { Física }\end{array}$} & \multicolumn{2}{|c|}{ Idade } & \multicolumn{2}{|c|}{ Peso } & \multicolumn{2}{|c|}{ IMC } & \multicolumn{2}{|c|}{$\% \mathrm{G}$} \\
\hline & & $r$ & $p$ & $r$ & $P$ & $r$ & $p$ & $r$ & $p$ \\
\hline \multirow{3}{*}{$\begin{array}{l}\text { Total } \\
(\mathrm{n}=363)\end{array}$} & FLEX & $-0,010$ & 0,925 & 0,026 & 0,618 & 0,024 & 0,650 & 0,023 & 0,688 \\
\hline & RML & $0,239 * *$ & 0,000 & 0,083 & 0,117 & $-0,088$ & 0,096 & $-0,420 * *$ & 0,000 \\
\hline & $\mathrm{ApC}$ & $0,269 * *$ & 0,000 & $0,179 * *$ & 0,001 & $-0,060$ & 0,316 & $-0,474 * *$ & $\mathbf{0 , 0 0 0}$ \\
\hline \multirow{3}{*}{$\begin{array}{l}\text { Meninas } \\
(n=188)\end{array}$} & FLEX & $-0,049$ & 0,508 & $-0,046$ & 0,335 & $-0,069$ & 0,350 & $-0,147^{*}$ & 0,045 \\
\hline & RML & 0,075 & 0,309 & $-0,164^{*}$ & 0,025 & $-0,181^{* *}$ & 0,013 & $-0,328 * *$ & $\mathbf{0 , 0 0 0}$ \\
\hline & ApC & $-0,033$ & 0,654 & $-0,107$ & 0,144 & $-0,150 *$ & 0,041 & $-0,143$ & 0,051 \\
\hline \multirow{3}{*}{$\begin{array}{l}\text { Meninos } \\
(n=175)\end{array}$} & FLEX & 0,056 & 0,465 & 0,167 & 0,128 & 0,129 & 0,090 & $-0,080$ & 0,917 \\
\hline & RML & $0,398 * *$ & 0,000 & 0,134 & 0,077 & $-0,055$ & 0,473 & $-0,330 * *$ & 0,000 \\
\hline & $\mathrm{ApC}$ & $0,507 * *$ & 0,000 & $0,184^{* * *}$ & 0,015 & $-0,070 *$ & 0,033 & $-0,475^{* *}$ & 0,000 \\
\hline
\end{tabular}

Legenda: FLEX: Flexibilidade; RML: Resistência Muscular Localizada; ApC: Aptidão Cardiorrespiratória. *Correlação significativa para $\mathrm{p} \leq 0,05 ; * *$ Correlação significativa para $\mathrm{p} \leq 0,01$.

Fonte: Autores.

\section{Discussão}

Ao analisar os resultados da correlação da adiposidade corporal com a aptidão física relacionada à saúde de escolares da rede pública de ensino, os principais achados desta pesquisa demonstram que quanto maior o \% menor o desempenho dos escolares nos testes motores referentes as capacidades físicas FLEX e RML no grupo feminino e, RML e ApC no grupo masculino. Dentre as capacidades físicas, a RML foi a mais afetada pelas elevadas taxas de adiposidade corporal, seguida por ApC e FLEX. Por outro lado, o IMC impactou negativamente a RML e ApC das meninas e, ApC dos meninos. Esses dados sugerem que embora as causas e consequências do sobrepeso e da obesidade sejam de conhecimento de toda a população, seus efeitos ainda impactam negativamente a aptidão física de muitas pessoas, especialmente jovens em idade escolar (Zimmermann \& Matheus, 2017).

Tão logo, nos últimos anos a quantidade de disfunções metabólicas entre crianças e adolescentes têm agravado consubstancialmente, algo preocupante, visto que a infância e a adolescência se mostram como momentos críticos para o desenvolvimento da obesidade (Monteiro et al., 2015; Oliveira \& Guedes, 2016; Simmonds et al., 2016).

Como prova disso, os resultados de $\% \mathrm{G}$ verificados em pesquisas atuais demonstram que nessa fase os escolares chegam muitas vezes a dobrar o peso corporal em poucos anos (Bloch et al., 2016; Carneiro et al., 2017). Malina et al. (2009) atribuem tal cenário as características biológicas e maturacionais que particularizam o período da adolescência. No entanto, muito além das questões genéticas, estes jovens refletem em suas escolhas a preferência da sociedade atual por hábitos compatíveis ao sedentarismo e ingestão de dietas hipercalóricas, que por sua vez, favorecem a instalação da obesidade e declínio da aptidão física (Paiva \& Costa, 2015; Bermejo-Cantarero et al., 2017; Freitas et al., 2020).

Assim, no que diz respeito ao $\% \mathrm{G}$ os resultados da presente pesquisa corroboram com o estudo de Pelegrini et al. (2015) realizado com 1.197 escolares de escola públicas, o qual encontrou valores médios de \%G de 16,7\% nos meninos e 
$26 \%$ nas meninas. Tais achados reforçam os resultados evidenciados por Burgos et al. (2010) que também identificaram \%G significativamente maior entre as meninas em relação ao grupo masculino de mesma idade.

Em continuação, com o propósito de analisarmos os efeitos da adiposidade corporal elevada na aptidão física dos escolares, verificamos uma correlação negativa significativa entre a FLEX e \%G na amostra feminina, que segundo Chumlhak et al. (2020) pode provocar a diminuição da qualidade de vida desses indivíduos em virtude do prognóstico de dores agudas e crônicas na região lombar e, maior incidência de desvios posturais, acendendo um alerta à respeito da ergonomia adotada pelos jovens durante os momentos de lazer e estudo.

Outra capacidade física que manifestou correlação negativa moderada com o \% tanto em meninos, quanto em meninas, foi a RML, algo já reportado nos estudos de Oliveira et al. (2017) e Rodrigues et al. (2018). Esses autores responsabilizam a inatividade física por tal cenário, pois esta favorece a diminuição da massa muscular e aumento da gordura corporal, principalmente na região abdominal (Oliveira et al., 2017; Rodrigues et al., 2018). Contudo, esta baixa RML pode causar problemas posturais, articulares, e até mesmo lesões músculo esqueléticas (Chumlhak et al., 2020), que podem interferir na execução de atividades da vida diária.

Por conseguinte, assim como Mello et al. (2013) em estudo realizado com 1.455 escolares de 10 à 17 anos, observamos que escolares com excesso de peso e adiposidade corporal, independentes do sexo e idade, possuem chance aumentada de apresentarem baixa ApC. A baixa ApC se caracteriza como um indicativo preocupante de saúde, que somada à obesidade, fruto da inatividade física e dieta rica em lipídeos e carboidratos, é capaz de provocar acometimentos futuros graves, como: doenças cardiovasculares, hipertensão arterial, diabetes melittus tipo 2, dislipidemias, entre outras patologias (Silva et al., 2010; Borfe et al., 2017; Rodrigues et al., 2018; Chumlhak et al., 2020).

Em linhas gerais, observa-se que apesar de parte dos escolares realizarem atividades físicas ao menos uma vez por semana, apenas uma pequena fração destes atinge os níveis recomendados de saúde em todas as variáveis estudadas (IMC, $\%$ G, FLEX, RML, ApC). Tal dizer corrobora com os resultados encontrados nos estudos de Pelegrini et al. (2015), Reis et al. (2017), Lima et al. (2018) e Chumlhak et al. (2020) os quais verificaram baixos índices de aptidão física em crianças e adolescentes em idade escolar em consequência da elevada taxa de adiposidade corporal.

Assim, visto que a obesidade atualmente atinge cerca de $20 \%$ da população brasileira (Brasil, 2019) e provoca um gasto anual ao cofres públicos de mais de 1 bilhão de reais somente no país (Nilson et al., 2019), mais do que avaliar é preciso analisar os dados de forma assertiva, atribuindo um olhar criterioso aos resultados não condizentes com os parâmetros de saúde, para que sejam traçadas intervenções que possam ajudar a diminuir os índices de obesidade infanto-juvenil, favorecendo o processo de desenvolvimento humano e transição para a vida adulta (Fermino et al., 2010; Pelegrini et al., 2015).

Por fim, indagamos que pesquisas futuras devem ser realizadas afim de acompanhar o estado de saúde e os índices de obesidade e aptidão física em escolares, uma vez que este estudo se limitou a avaliação aguda das características físicas de escolares do ensino público, não considerando os aspectos motivacionais e socioeconômicos, bem como escolares do ensino privado. No entanto, cabe ressaltar que este estudo seguiu rigorosamente seu objetivo ofertando resultados atuais para intervenções pontuais e análises futuras dessa população que compreende a maior parcela dos escolares do país.

\section{Conclusão}

Com base nos resultados encontrados conclui-se que quanto maior o $\% \mathrm{G}$ menor eram o desempenho dos escolares nos testes motores referentes as capacidades físicas estudadas. Portanto, a obesidade nesses indivíduos se caracteriza como fator preponderante à baixa aptidão física. Algo preocupante visto que estes fatores podem favorecer o surgimento e agravamento de 
Research, Society and Development, v. 10, n. 3, e52310313680, 2021

(CC BY 4.0) | ISSN 2525-3409 | DOI: http://dx.doi.org/10.33448/rsd-v10i3.13680

comorbidades, prejudicando o processo de desenvolvimento humano e início da vida adulta. Finalmente, incentivamos o desenvolvimento de estudos futuros para que haja melhor esclarecimento sobre o comportamento das variáveis estudadas, conscientização dos efeitos da obesidade e adoção de um estilo de vida saudável. Assim, sugerimos aos futuros estudos a ampliação amostral, inserção de escolares de instituições privadas, e a investigação de aspectos socioeconômicos, nutricionais e motivacionais que possam inferir sobre a saúde dos escolares brasileiros.

\section{Agradecimentos}

A Secretaria Municipal da Educação de Votuporanga/SP, Brasil, pela colaboração e livre acesso aos estabelecimentos escolares, ao Centro Universitário de Votuporanga - UNIFEV, Votuporanga/SP, Brasil, pelo apoio financeiro e material concedido durante a realização desta pesquisa, e aos voluntários por sua disponibilidade e interesse em contribuir com o ensino e à pesquisa.

\section{Referências}

Bermejo-Cantarero, A., Álvarez-Bueno, C., Martinez-Vizcaino, V., García-Hermoso A., Torres-Costoso A. I., \& Sánchez-López M. (2017). Association between physical activity, sedentary behavior, and fitness with health related quality of life in healthy children and adolescents: A protocol for a systematic review and meta-analysis. Medicine, 96(12), e6407. https://doi.org/ 10.1097/MD.0000000000006407

Bloch, K. V., Klein, C. H., Szklo, M., Kuschnir, M. C. C., Abreu, G. D. A., Barufaldi, L. A., ... \& Goldberg, T. B. L. (2016). ERICA: prevalências de hipertensão arterial e obesidade em adolescentes brasileiros. Revista de Saúde Pública, 50(suppl1), 9s. https://doi.org/10.1590/S01518-8787.2016050006685

Borfe, L., Rech, D. C., Benelli, T. E., Paiva, D. N., Pohl, H. H., \& Burgos, M. S. (2017). Association between childhood obesity and cardiorespiratory fitness: a systematic review. Revista Brasileira em Promoção da Saúde, 30(1), 118-24. http://doi.org/10.5020/18061230.2017.p118

Brasil. Ministério da Saúde. Secretaria de Vigilância em Saúde. Departamento de Análise em Saúde e Vigilância de Doenças não Transmissíveis. (2019). Vigitel Brasil 2018: vigilância de fatores de risco e proteção para doenças crônicas por inquérito telefônico: estimativas sobre frequência e distribuição sociodemográfica de fatores de risco e proteção para doenças crônicas nas capitais dos 26 estados brasileiros e no Distrito Federal em 2018. Brasília: Ministério da Saúde. http://bvsms.saude.gov.br/bvs/publicacoes/vigitel_brasil_2018_vigilancia_fatores_risco.pdf

Burgos, M. S., Reuter, C. P., Burgos, L. T., Pohl, H. H., Pauli, L. T. S., Horta, J. A., ... \& Camargo, M. (2010). Uma análise entre índices pressóricos, obesidade e capacidade cardiorrespiratória em escolares. Arquivos Brasileiros de Cardiologia,94(6), 788-793. https://doi.org/10.1590/S0066$782 \mathrm{X} 2010005000046$

Carneiro, C. D. S., Peixoto, M. D. R. G., Mendonça, K. L., Póvoa, T. I. R., Nascente, F. M. N., Jardim, T. D. S. V., ... \& Jardim, P. C. B. V. (2017). Excesso de peso e fatores associados em adolescentes de uma capital brasileira. Revista Brasileira de Epidemiologia, 20(2), 260-273. https://doi.org/10.1590/19805497201700020007

Castro, J. A. C., Lima, T. R., \& Silva, D. A. S. (2018). Body composition estimation in children and adolescents by bioelectrical impedance analysis: A systematic review. Journal of bodywork and movement therapies, 22(1), 134-146. http://doi.org/10.1016/j.jbmt.2017.04.010

Chumlhak, Z., Oliveira, V. M., Brasil, M. R., Mattes, V. V., Menegaldo, P. H. I., \& da Silva, S. R. (2020). Nível de aptidão física, imagem corporal e desempenho escolar em escolares de séries iniciais do ensino fundamental. Research, Society and Development,9(7), e178973558-e178973558. http://doi.org/10.33448/rsd-v9i7.3558

Costa, E. A., Faria, V. A., Gallo, I. S., Brigatto, F. A., de Oliveira, T. P., \& Junior, A. C. T. (2020). Relação entre testes de aptidão física, índice de massa corporal e relação cintura estatura em escolares das redes públicas e privadas. Brazilian Journal of Development, 6(12), $102486-102497$. http://doi.org/10.34117/bjdv6n12-665

Faria, E. R. D., Gontijo, C. A., Franceschini, S. D. C. C., Peluzio, M. D. C. G., \& Priore, S. E. (2014). Composição corporal e risco de alterações metabólicas em adolescentes do sexo feminino. Revista Paulista de Pediatria, 32(2), 207-215. http://doi.org/10.1590/S0066-782X2009000800009

Fermino, R. C., Rech, C. R., Hino, A. A. F., Rodriguez Añez, C. R., \& Reis, R. S. (2010). Atividade física e fatores associad os em adolescentes do ensino médio de Curitiba, Brasil. Revista de Saúde Pública, 44(6), 986-995. https://doi.org/10.1590/S0034-89102010000600002

Freitas, A. S., De Góes, A. V. S., Mendes, J. C. L., Deusdará, F. F., De Barros, P. E. G., Durães, G. M., \& Rodrigues, V. D. (2020). Obesidade na adolescência: IMC, circinferência abdominal e aptidão física em escolares do Norte de Minas. RBONE - Revista Brasileira de Obesidade, Nutrição e Emagrecimento, 14(84), 37-45. http://www.rbone.com.br/index.php/rbone/article/view/1144/930

Gaya, A. C. A., \& Gaya, A. R. (2016). Projeto Esporte Brasil PROESP-Br. Manual de testes e avaliação, 1-26. https://www.ufrgs.br/proesp/index.php

Hopkins, W., Marshall, S., Batterham, A., \& Hanin, J. (2009). Progressive statistics for studies in sports medicine and exercise science. Medicine Science in Sports Exercise, 41(1), 3-12. http://doi.org/10.1249/MSS.0b013e31818cb278. 
Research, Society and Development, v. 10, n. 3, e52310313680, 2021

(CC BY 4.0) | ISSN 2525-3409 | DOI: http://dx.doi.org/10.33448/rsd-v10i3.13680

Instituto Brasileiro de Geografia e Estatística (2017). População no último censo, panorama de Votuporanga. https://cidades.ibge.gov.br/brasil/sp/votuporanga/panorama

Lima, F. É. B., Coco, M. A., Pellegrinoti, I. L., Lima, W. F., Da Silva-Lima, S. B., \& Lima, F. B. (2018). Aptidão física relacionada ao desempenho motor e a saúde de adolescentes da região sudoeste do estado de São Paulo e norte pioneiro do estado do Paraná. RBONE - Revista Brasileira de Obesidade, Nutrição e Emagrecimento, 12(75), 908-919. http://www.rbone.com.br/index.php/rbone/article/view/812/609

Lopes, V. P., Malina, R. M., Gomez-Campos, R., Cossio-Bolaños, M., Arruda, M. D., \& Hobold, E. (2019). Índice de massa corporal e aptidão física em adolescentes brasileiros. Jornal de Pediatria, 95(3), 358-365. https://doi.org/10.1016/j.jped.2018.04.003

Malina, R. M., Bouchard, C., \& Bar-Or, O. (2009). Crescimento, maturação e atividade física. São Paulo: Phorte.

Mello, J. B., Da Silva Hernandez, M., Farias, V. M., Dos Santos Pinheiro, E., \& Bergmann, G. G. (2015). Aptidão física relacionada ao desempenho motor de adolescentes de Uruguaiana, Rio Grande do Sul. Revista Brasileira de Ciência e Movimento, 23(4), 72-79. http://doi.org/10.18511/0103-1716/rbcm.v23n4p7279

Mello, J. B., Da Silva Ribeiro, Y., Castagna, A., de Araújo Bergmann, M. L., \& Bergmann, G. G. (2013). Baixa aptidão cardiorrespiratória está associada ao excesso de peso em crianças e adolescentes independente do sexo e da idade. Revista Brasileira de Ciência e Movimento,21(4), 56-62. http://doi.org/10.18511/0103-1716/rbcm.v21n4p56-62

Mendonça, C. P., \& Anjos, L. A. D. (2004). Fatores dietéticos e de atividade física como determinantes do aumento do sobrepeso / obesidade no Brasil. Cadernos de Saúde Pública, 20(3), 698-709. https://doi.org/10.1590/S0102-311X2004000300006

Meneguci, J., Santos, D. A. T., Silva, R. B., Santos, R. G., Sasaki, J. E., Tribess, S., ... \& Virtuoso Jr, J. S. (2015). Sedentary behavior: concept, physiological implications and the assessment procedures. Motricidade, 11(1), 160-175. http://doi.org/10.6063/motricidade.3178

Monteiro, A. B., Lopes, G. O., Bustos, P. E., Ramos, M. R., Santos, T. R., Vidal, V. F., ... \& Figueiredo, T. C. (2015). Aptidão física e composição corporal de alunos do ensino fundamental da rede pública em Jacarepaguá-RJ. RBPFEX - Revista Brasileira de Prescrição e Fisiologia do Exercício, 9(55), 485-496. http://doi.org/10.1249/1981-9900

Nahas, M. V. (2017). Atividade física, saúde e qualidade de vida: conceitos e sugestões para um estilo de vida ativo. (7a ed). Londrina: Midiograf.

Nascimento, M. B., Porto, M. J., Souza, J. P., Guerra, M. F. S., Araujo, A. M. B., Andrade, W. B., Santana, W. N. B., Santos, G. P., Santana, A. F., \& Silva, S. R. S. (2021). Adolescent obesity: a profile currently outlined through narrative review. Research, Society and Development, 10(1), e26710111857. https://doi.org/10.33448/rsd-v10i1.11857

Nilson, E. A. F., Andrade, R. D. C. S., Brito, D. A. D., \& Oliveira, M. L. D. (2020). Custos atribuíveis a obesidade, hipertensão e diabetes no Sistema Único de Saúde, Brasil, 2018. Revista Panamericana de Salud Pública, 44, e32. https://doi.org/10.26633/RPSP.2020.32

Oliveira, R. G. D., \& Guedes, D. P. (2016). Atividade física, comportamento sedentário, aptidão cardiorrespiratória e síndrome metabólica em adolescentes: revisão sistemática e meta-análise de evidências observacionais. PloS One, 11(12), e0168503. https://doi.org/ 10.1371/journal.pone.0168503

Oliveira, V. M., Brasil, M. R., Chumlhak, Z., Cordel, P. T., Czuy, G. H. B., \& da Silva, S. R. (2017). Nível de aptidão física em escolares: Influência do índice de massa corporal, sexo e quantidade de sono. Saúde e Meio Ambiente: revista interdisciplinar, 6(1), 4-17. http://doi.org/10.24302/sma.v6i1.1382

Paes, S. T., Marins, J. C. B., \& Andreazzi, A. E. (2015). Efeitos metabólicos do exercício físico na obesidade infantil: uma visão atual. Revista Paulista de Pediatria, 33(1), 122-129. https://doi.org/10.1016/j.rpped.2014.11.002

Paiva, N. M. N., \& Costa, J. (2015). A influência da tecnologia na infância: desenvolvimento ou ameaça. Psicologia. pt, 1, 1-13. https://www.psicologia.pt/artigos/textos/A0839.pdf

Pelegrini, A., Silva, D. A. S., De Lima Silva, J. M. F., Grigollo, L., \& Petroski, E. L. (2015). Indicadores antropométricos de obesidade na pred ição de gordura corporal elevada em adolescentes. Revista Paulista de Pediatria, 33(1), 56-62. http://doi.org/10.1016/j.rpped.2014.06.007

Pereira, A. S., Shitsuka, D. M., Parreira, F. J., \& Shitsuka, R. (2018). Metodologia da pesquisa científica. [e-book]. Santa Maria: UAB/NTE/UFSM. https://repositorio.ufsm.br/bitstream/handle/1/15824/Lic_Computacao_Metodologia-Pesquisa-Cientifica.pdf?sequence=1.

Pereira-Lancha, L. O., Campos-Ferraz, P. L., \& Lancha, A. H. (2012). Obesidade: considerações sobre etiologia, metabolismo e uso de modelos experimentais. Diabetes, síndrome metabólica e obesidade: alvos e terapia, 5, 75-87. https://doi.org/ 10.2147/DMSO.S25026

Petroski, E. L., Silva, A. F. D., Rodrigues, A. B., \& Pelegrini, A. (2011). Aptidão física relacionada a saúde em adolescentes brasileiros residentes em áreas de médio/baixo índice de desenvolvimento humano. Revista de Salud Pública, 13(2), 219-228. https://doi.org/ article/rsap/2011.v13n2/219-228

Reis, M. S., Lisboa, T., Alexandre, J. M., Capistrano, R., \& Beltrame, T. S. (2017). Aptidão cardiorrespiratória associada ao índice de massa corporal em escolares entre 7 a 10 anos de idade. RBPFEX - Revista Brasileira de Prescrição e Fisiologia do Exercício, 11(64), 122-127. http://www.rbpfex.com.br/index.php/rbpfex/article/view/1088/891

Rodrigues, G. C., Marques, K. C., De Castro Silveira, J. F., De Borba Schneiders, L., Burgos, L. T., \& Reuter, C. P. (2018). Obesidade e aptidão física relacionada à saúde: um estudo com escolares de 10 a 13 anos de Santa Cruz do Sul-Brasil. Arquivos de Ciências da Saúde,25(3), 60-63. https://doi.org/10.17696/2318-3691.25.3.2018.1276

Santos, G. E. O. (2017). Cálculo amostral: calculadora on-line. http://www.calculoamostral.vai.la 
Research, Society and Development, v. 10, n. 3, e52310313680, 2021

(CC BY 4.0) | ISSN 2525-3409 | DOI: http://dx.doi.org/10.33448/rsd-v10i3.13680

Secretaria Municipal da Educação de Votuporanga (2017). Número de alunos matriculados. http://votuporanga.sp.gov.br/n/publicacao/?x=educacaoculturaeturismo\&p=20166782856-numero-de-alunos-matriculados.

Silva, S. P. D., Santos, A. C. D. S., Silva, H. M. D., Costa, C. L. A., \& Nobre, G. C. (2010). Aptidão cardiorespiratória e composição corporal em crianças e adolescentes. Motriz: Revista de Educação Física, 16(3), 664-671. http://doi.org/10.5016/1980-6574.2010v16n3p664

Simmonds, M., Llewellyn, A., Owen, C. G., \& Woolacott, N. (2016). Predicting adult obesity from childhood obesity: a systematic review and meta-analysis. Obesity reviews, 17(2), 95-107. http://doi.org/10.1111/obr.12334.

Slaughter, M. H., Lohman, T. G., Boileau, R., Horswill, C. A., Stillman, R. J., Van Loan, M. D., \& Bemben, D. A. (1988). Skinfold equations for estimation of body fatness in children and youth. Human biology, 60(5), 709-723. https://www.jstor.org/stable/41464064?seq=1

Thomas, J. R., Nelson, J. K., \& Silverman, S. J. (2012). Métodos de pesquisa em atividade física. (6a ed). Porto Alegre: Artmed.

Zawadzki, D., Stiegler, N. D. F. F., \& Brasilino, F. F. (2019). Aptidão e atividade física relacionados à saúde de adolescentes entre 11 e 14 anos. RBPFEX Revista Brasileira de Prescrição e Fisiologia do Exercício, 13(83), 444-453. http://www.rbpfex.com.br/index.php/rbpfex/article/view/1683/1215

Zimmermann, A. M., \& Matheus, S. C. (2017). Associação entre estado nutricional, aptidão física e imagem corporal de crianças e adolescentes. RBNE Revista Brasileira de Nutrição Esportiva, 11(64), 516-525. http://www.rbne.com.br/index.php/rbne/article/view/850/658 